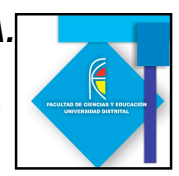

\title{
EL EXPERIMENTO DE MICHELSON Y MORLEY: UNA PROPUESTA DIDACTICA PARA LA ENSEÑANZA DE LA RELATIVIDAD ESPECIAL ${ }^{1}$
}

\section{THE EXPERIMENT OF MICHELSON AND MORLEY: A DIDACTIC PROPOSAL FOR TEACHING OF SPECIAL RELATIVITY}

\author{
Adriana Carolina Díaz López ${ }^{2}$
}

\begin{abstract}
Resumen
El presente documento de corte histórico- crítico, muestra un estudio sobre los experimentos de Michelson y Morley (1881-87) mediante la lectura de los artículos originales y algunos textos de Física universitarios, desde los cuales se han caracterizado las problemáticas inmersas en el contexto del siglo XIX que apoyaron su realización, las hipótesis de base, sus elementos de construcción y las conclusiones respectivas. Se proponen algunas reflexiones respecto a las interpretaciones que presentan los libros de texto, su contribución a finales del siglo XIX al concepto de marco de referencia con la idea del éter y finalmente, los criterios de una propuesta didáctica para un curso de relatividad Especial, destacando el uso de la historia en los contextos de enseñanza de la Física.
\end{abstract}

Palabras clave: Historia y filosofía de las ciencias, enseñanza de la física, Éter luminífero, experimento de Michelson y Morley (M-M), Teoría especial de la Relatividad, marco de referencia privilegiado, Didáctica de las ciencias.

\begin{abstract}
This document of a historical-critical method, shows a study of the Michelson-Morley experiment (1881-1887) by reading the original papers and college physics texts, from which we have characterized the problems embedded in the context nineteenth century that supported their implementation, the baseline scenario, building components and their respective conclusions. We propose some reflections on the interpretations that have textbooks, their contribution to late nineteenth century the concept of frame of reference with the idea of ether, and finally, the criteria of an educational proposal for a course in special relativity, emphasizing the use of history in the contexts of physics education.
\end{abstract}

Trabajo de Grado de DIAZ, ADRIANA (2009).Departamento de Física, centro de Documentación, Universidad Pedagógica Nacional. 1981 astroadri@hotmail.com 


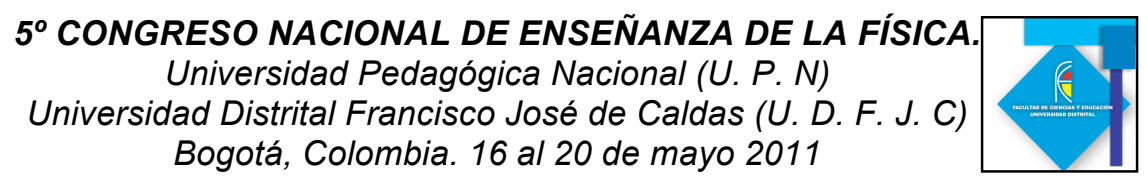

Keywords: History and philosophy of science, physics education, luminiferous ether, Michelson-Morley experiment (MM), Special Theory of Relativity, privileged framework, Science education.

\section{Introduccion}

El experimento de Michelson y Morley se ha constituido en una fuente de debate por su "crucialidad" o contribución a los orígenes de la Teoría Especial de la Relatividad (TER), dadas las múltiples interpretaciones que algunos textos muestran respecto a su finalidad y conclusiones como por ejemplo: "El experimento proporcionó una clara prueba de que no existe ningún éter y de que la velocidad de la luz es constante independientemente del movimiento de la fuente" (COUGHLAN y DODD, 1991, p. 9 en CASSINI. A Y LEVINAS. L., 2005,). En un sentido pedagógico, el experimento suele presentarse en los cursos de Relatividad Especial de manera descriptiva sin hacer énfasis en su desarrollo y posibles implicaciones en los orígenes de la TER. No se analizan las condiciones históricas que rodearon los experimentos de 1881 y 1887 , no se reconoce las críticas propuestas ni su contribución en la caracterización del concepto de marco de referencia a partir del éter, idea que por cierto fue significativa a la luz de explicar el movimiento absoluto desde la relatividad galileana (principio de adición de velocidades).

Estos presupuestos dan cuenta de una falta de claridad sobre el experimento en los contextos de enseñanza, en tanto la historia no aparece como un elemento que el maestro emplea para hacer énfasis en la actividad científica ligada a los contextos en los que se desarrolla, mas bien suele mostrarse como un recurso oportuno, cronológico y anecdótico, pero no se referencia en esa búsqueda de dialogar con los autores y mirar los textos de manera reflexiva. Si involucramos la historia dentro del contexto de la enseñanza de la Relatividad asumiendo el experimento como un elemento de reflexión frente a las problemáticas abordadas en un momento, es posible confrontar los procesos de construcción de conocimiento en el aula y las proposiciones filosóficas que suelen contemplarse en los textos de carácter universitario. Considerando entonces el experimento de Michelson y Morley como un antecedente a la Teoría Especial de la Relatividad - TER y un elemento que permite construir una propuesta didáctica para la enseñanza de la Relatividad, surgen dos preguntas:

¿En qué medida el experimento de Michelson y Morley contribuye a la caracterización del concepto de marco de referencia en el paso de la física clásica a la teoría especial de la relatividad?

¿Cómo los estudios en historia de las ciencias pueden contribuir a comprender esta problemática y aportar al desarrollo de propuestas didácticas para la enseñanza de la relatividad frente a los procesos de construcción de conocimiento que se imparten en el aula?

A partir del estudio de los documentos: "The relative motion of the earth and the luminiferous ether (Albert Michelson, American Journal of science. Serie 3, XXII, Pp.12029,1881 )" y "On the relative motion of the earth and the luminiferous ether" (A. A. Michelson y E. W. Morley, American Journal of Science. Serie 3, XXXIV, Pp.333-45, 1887), 


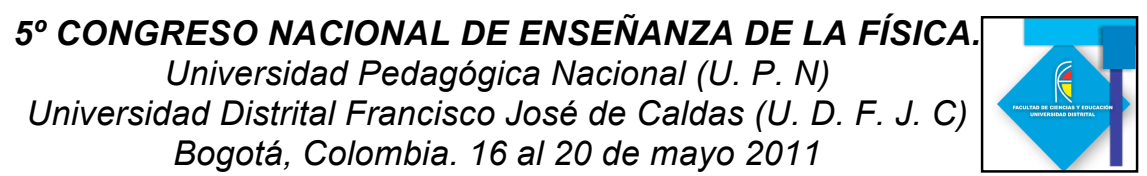

se identificaron las condiciones que rodearon la realización del experimento y se hizo una caracterización de los intentos realizados en los años de 1881 y 1887. Con base en ello y

en la revisión de algunos textos de Física Universitarios de autores se hizo una reflexión sobre la presentación del experimento, esto permitió obtener algunas herramientas para la elaboración del módulo didáctico.

\section{Una mirada histórica al experimento Antecedentes al experimento}

Situados en un contexto histórico, el experimento de Michelson y Morley, surge a finales del siglo XIX, donde las diversas imágenes del éter que los científicos construyeron lograban justificar los diferentes fenómenos físicos, como el caso de la hipótesis de arrastre parcial para explicar la aberración estelar y el éter electromagnético como soporte de los fenómenos ópticos y electromagnéticos. De igual manera, la determinación de la velocidad de la luz y trabajos como el postulado por J.C. Maxwell basados en la determinación de la velocidad del sistema solar con respecto al éter (FRENCH,1974), mostraron una iniciativa para plantear métodos que permitieran medir el movimiento de los cuerpos respecto al espacio absoluto en el que el éter resultaba como marco de referencia privilegiado. Partiendo de estos supuestos, Michelson realiza su experimento (1881) para medir la velocidad de la tierra respecto al éter con base en los cálculos de los tiempos registrados por dos haces de luz que se interferían en lugar de una sola trayectoria como la consideraba Maxwell. De acuerdo al diagrama de interferencia presentado se tendría a consideración el efecto del éter sobre la tierra (Ver figura 1). Críticas por parte de Lorentz y otros científicos llevó a Michelson a repetir su experimento con Morley en 1887.

\section{Los experimentos de 1881 y 1887}

Al realizar las observaciones el trabajo no fue posible debido a la extrema sensibilidad del instrumento (por las vibraciones externas) que afectaba las franjas, además de los errores teóricos planteados por Potier en 1881 que identificaban el pasar por alto la influencia del movimiento de la tierra en el sendero vertical. Teniendo en cuenta el mismo funcionamiento del interferómetro pero otros detalles de fabricación (MICHELSON. A y MORLEY. E, 1887), los autores repitieron el experimento en Cleveland hacia el año de 1887. Consideraron esta vez la influencia del movimiento orbital terrestre en el brazo vertical: supusieron que si el aparato se movía en la dirección con la velocidad orbital de la tierra, ahora la dirección en la que se movían los rayos se

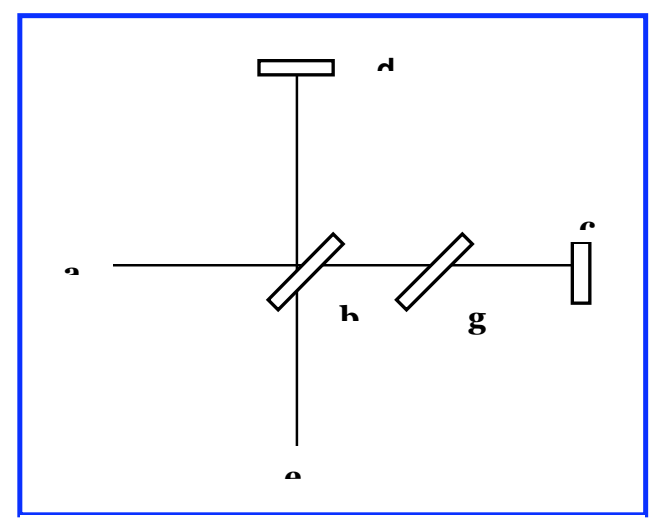

Figura 1. Diseño del Interferómetro de Michelson (1881). Dos rayos de luz que viajan en caminos perpendiculares se reflejan e interfieren mostrando un espectro de interferencia. La diferencia de tiempos evidenciada en el corrimiento de las franjas permitiría dar cuenta de los efectos del éter. alteraba, de tal manera que el rayo reflejado verticalmente era afectado por los efectos de aberración. 


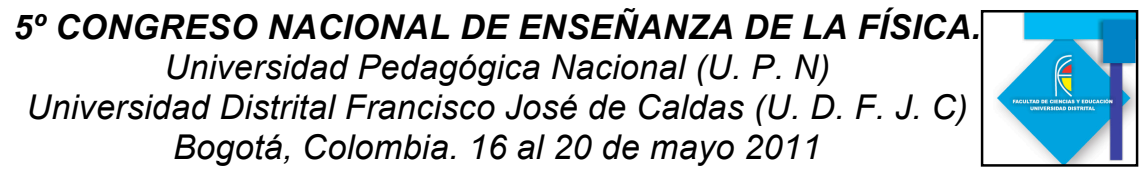

Las conclusiones a las que llegaron los autores no indican la inexistencia del éter: "...Parece lícito concluir en caso de que existiese algún desplazamiento debido al movimiento relativo de la tierra y el éter luminífero, dicho desplazamiento no podría ser superior a 0.01 veces la separación entre las franjas", ya que el desplazamiento esperado era de 0.4 franjas (MICHELSON. A y MORLEY. E, 1887). Debido a estos resultados negativos en el sentido de no observar un desplazamiento de las franjas perceptible y de acuerdo a lo esperado, algunos físicos teóricos empezaron la búsqueda de posibles explicaciones como Fitzgerald (1892) y Lorentz (1895) quienes habían seguido de cerca el trabajo de Michelson. Al respecto, propusieron que el éter sigue siendo considerado en reposo en el espacio absoluto pero cualquier cuerpo que se mueva con respecto a este, se contrae en la dirección de su movimiento, de modo que el brazo que se mueve paralelamente al éter sufre una contracción en su longitud en un factor de $\sqrt{1 \square \frac{v^{2}}{c^{2}}}$ y puede justificarse el que no exista diferencia de trayectoria al girar el aparato.

\section{Reflexiones sobre la presentacion del experimento en los libros de texto}

La imagen distorsionada del experimento parte de la propuesta que los libros de texto han presentado, y por consiguiente del uso que los docentes universitarios hacen de estos para construir su explicación en el aula. Teniendo en cuenta este supuesto se realizó un estudio con seis libros de texto: FÍsICA. Serway, Raymond A. Editorial Mac Graw- Hill. México. Cuarta edición, Vol.2, 1999; CONCEPTOS DE FÍSICA. Hewitt, Paul. G. Editorial Limusa, S. A, Grupo Noriega Editores, México. D. F, Décima reimpresión, 2001; FÍSICA. Tipler, Paul. A. Tercera edición. Editorial Reverte, S. A. Barcelona, España, 1996; FISICA. Sears Francis W. Semansky, Mark. W. y Young, Hugh. Undécima edición. Editorial Pearson. Educación de México, S. A, 2004; FÍSICA. Sears, Francis W. Cuarta edición. Madrid, Aguilar 1978, FISICA UNIVERSITARIA, Sears, Francis W. y Semansky, 1998. De estos es posible concluir que en algunos de ellos, se hace una presentación del modelo del interferómetro de 1881 para introducirlo como un objeto de aplicación al fenómeno de interferencia sin hacer énfasis en su contexto histórico, desconociendo las falencias de diseño y resultados experimentales que llevaron a un nuevo planteamiento del mismo en 1887 (Ver tabla 1). Se da a entender que Michelson diseñó su experimento en 1881 pero que su famosa aplicación y resultados negativos fueron obtenidos con Morley seis años después. 


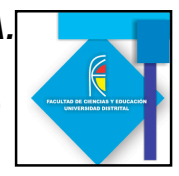

\begin{tabular}{|c|c|c|c|c|c|}
\hline Bibliografia & $\begin{array}{l}\text { Sección de } \\
\text { presentación }\end{array}$ & Antecedentes & $\begin{array}{l}\text { Caracterización } \\
\text { de los } \\
\text { montajes }\end{array}$ & $\begin{array}{l}\text { Prediccion } \\
\text { de los } \\
\text { resultados }\end{array}$ & Conclusiomes \\
\hline $\begin{array}{l}\text { Sears, } \\
\text { Francis W. y } \\
\text { Semansky. } \\
1998\end{array}$ & $\begin{array}{l}\text { Pág. 1154, } \\
\text { Cáp.37: } \\
\text { Interferencia }\end{array}$ & $\begin{array}{l}\text { Idea del éter } \\
\text { como medio de } \\
\text { transporte de } \\
\text { las ondas } \\
\text { luminosas. No } \\
\text { presentan mas } \\
\text { datos al } \\
\text { respecto }\end{array}$ & $\begin{array}{l}\text { Descripción } \\
\text { interferómetro } \\
\text { para mostrar su } \\
\text { aplicabilidad en } \\
\text { el fenómeno de } \\
\text { interferencia. } \\
\text { Montaje de } 1881 \\
\text { sin hacerlo } \\
\text { explícito. }\end{array}$ & $\begin{array}{l}\text { Aunque se } \\
\text { propone el } \\
\text { montaje de } \\
1881 \text { sin } \\
\text { hacer la } \\
\text { aclaración, } \\
\text { no se hace } \\
\text { referencia a } \\
\text { la } \\
\text { predicción } \\
\text { de los } \\
\text { resultados } \\
\text { en ese año, } \\
\text { otorgándole } \\
\text { mayor } \\
\text { importancia } \\
\text { y } \\
\text { conclusión } \\
\text { al de 1887. }\end{array}$ & $\begin{array}{l}\text { Interpretación } \\
\text { resultado nulo } \\
\text { asumiendo a la } \\
\text { tierra en } \\
\text { reposo, } \\
\text { incongruente } \\
\text { con el valor de } \\
\text { los originales y } \\
\text { con la } \\
\text { apreciación de } \\
\text { los autores } \\
\text { frente a un } \\
\text { pequeño } \\
\text { movimiento } \\
\text { relativo entre } \\
\text { la tierra } \\
\text { respecto al } \\
\text { éter: } \\
\text { "El } \\
\text { desplazamiento } \\
\text { observado, fue } \\
\text { de } \\
\text { exactamente } \\
\text { cero". }\end{array}$ \\
\hline
\end{tabular}

Tabla 1

El análisis completo se encuentra en DIAZ, ADRIANA (2009). El experimento de Mihelson y Morley: Una propuesta didáctica para la enseñanza de la Relatividad Especial. Universidad Pedagógica Nacional, Departamento de física. Anexo 7. Pág. xxiii.

La interpretación de "resultado nulo" parece indicar ausencia de algún desplazamiento de las franjas de interferencia lo que resulta inconsistente con los resultados obtenidos. Lo que podría afirmarse es que los resultados eran negativos dado que los desplazamientos 


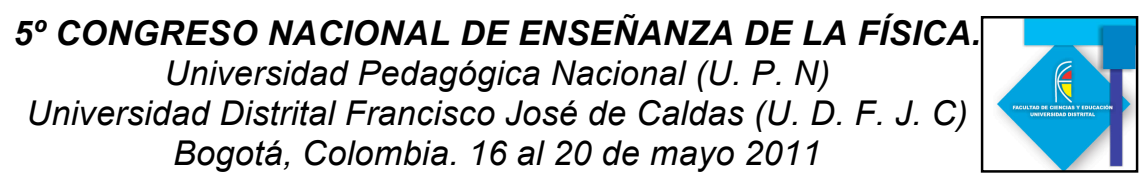

se aproximaron a cero y estaban fuera de los límites teóricos

propuestos. Por otro parte, las conclusiones a las que llegaron M-M parecen ser muy apresuradas en cuanto al planteamiento de algunas premisas sobre las cuales no se tenía un conocimiento muy amplio y a la ausencia de algunos elementos asociados a la actividad experimental.

\section{Los estudios histórico-críticos como forma de construir opciones para la enseñanza de la física}

Los estudios histórico críticos como procesos de recontextualización de saberes y el análisis de los libros de texto nos llevan a formular una propuesta didáctica soportada desde varios criterios, el primero corresponde a la lectura de los originales que el estudiante debe hacer para comprender el contexto histórico y las apreciaciones reales de los autores para el diseño, en segundo lugar la producción de significados desde el ejercicio de contraste con los libros de texto y finalmente la discusión a través de diferentes técnicas de comunicación o dinámicas de grupo de los participantes en gran grupo que permita retroalimentar y construir el conocimiento. La propuesta elaborada y soportada en el trabajo de grado consta de tres fases y una cartilla anexa con orientaciones al docente para realizar las sesiones y aplicar el trabajo a estudiantes de un curso de Relatividad Especial en el nivel universitario.

\section{Conclusiones}

La reflexión que los textos hacen frente a los experimentos, refleja por parte de algunos una interpretación propia de los originales pues se pasa por alto aspectos del primer experimento y en muchas ocasiones no existe claridad en la finalidad y conclusiones a las que sus autores llegaron, como el caso de afirmar que sus resultados probaron la inexistencia del éter o su crucialidad frente a la Teoría Especial de la Relatividad sin recurrir a alguna postura o argumentos válidos para justificar esta clase de afirmaciones. Ante este hecho, los estudios históricos se constituyen en una fuente de comprensión y análisis de la actividad científica del momento y de las condiciones que rodearon el experimento en esa búsqueda de resolver problemáticas lo cual permite hacer una lectura diferente de los textos y proponer criterios que permitan ver el experimento con nuevos ojos para proveer de elementos al maestro al momento de enseñarlo.

\section{Referencias Bibliográficas}

AYALA, María Mercedes (2005). Los análisis histórico-críticos y la recontextualización de saberes científicos, grupo Física y Cultura, Departamento de Física, Universidad Pedagógica Nacional, Bogotá.

CASSINI, Alejandro y LEVINAS, Leonardo.(2005).La reinterpretación del experimento de Michelson Morley por la relatividad Especial. Scientle studia, Sao Paulo, V.3, n.4, Pp. 547 81.

MUNERA, H.A. (2007). El experimento de Michelson y Morley y el segundo postulado de Einstein: inextricablemente unidos. Departamento de Física, Universidad Nacional de Colombia, Bogotá, Colombia. 